\title{
SALT - A Cost Effective Science Machine: Statistical evaluation of publications
}

\author{
A.C. Schröder* \\ South African Astronomical Observatory, P.O. Box 9 Observatory, Cape Town, South Africa; \\ Southern African Large Telescope, P.O. Box 9 Observatory, Cape Town, South Africa \\ E-mail: anja@saao.ac.za \\ P. Väisänen \\ South African Astronomical Observatory, P.O. Box 9 Observatory, Cape Town, South Africa; \\ Southern African Large Telescope, P.O. Box 9 Observatory, Cape Town, South Africa
}

\section{S.M. Crawford}

South African Astronomical Observatory, P.O. Box 9 Observatory, Cape Town, South Africa; Southern African Large Telescope, P.O. Box 9 Observatory, Cape Town, South Africa

\begin{abstract}
The evaluation of SALT publications is an important means for successful science operations of the observatory. Presented here are some of the more interesting statistics based on information from 121 refereed publications as well as from the SALT observing programs proposals used in these publications. These statistics, however, need to be taken with a grain of salt: they reflect clearly the long start-up/commissioning phase until the problems with the image quality had been sorted out, that is, proper SALT observations started only in 2011. That means there are less than 4 years of full science operations available, and with a $\sim 2$-year delay until publication only about 2 years are useful for unbiased statistics. On the other hand, the data since 2011 show a rise in publication rate comparable to that of other large observatories and indeed demonstrate that SALT is most cost effective. The citation rate per paper up to 2014 is 15.7 which compares well with that of other major telescopes. Of interest is also that these early science publications show a predominance for single-target, long-slit data which take the least time to reduce.
\end{abstract}

SALT Science Conference 2015 -SSC2015,

1-5 June 2015

Stellenbosch Institute of Advanced Study, South Africa

${ }^{*}$ Speaker. 


\section{Introduction}

The publication rate of science done with a particular observatory is often the best indicator of the efficiency and impact of an observatory (e.g., [1], [2], [3]). However, it is equally important to understand what goes into these science papers and what does not. The observatory can use this feedback to improve in various ways, e.g., improving the selection of instruments, offering more specific user support, or passing on this feedback to the TAC to improve priority settings.

While SALT has started full science operations only four years ago and with the typical delay between observations and publications, we have only a couple of years to work with. Yet, understanding what particularly drives the early science output of an observatory can also help future observatories to set priorities for the first couple of years of operations.

In this paper, we present the statistics derived from 121 refereed publications as described in Sec. 2. The statistics in Sec. 3 range from the publication and citation rate over statistics per paper ('who publishes?' Sec. 3.1) to statistics per proposal ('what is the most productive input?' Sec. 3.2). We give conclusions of the findings in Sec. 4.

\section{Paper selection}

The following criteria were used to select the SALT publications:

- Select all refereed papers on ADS that have the word SALT in it;

- Check each paper to verify if indeed it is about SALT and classify these in the following way:

- It presents and analyses SALT observations (labelled 'data');

- It describes planned or ongoing SALT science projects (labelled 'general science');

- It describes the telescope, instrument or reduction/analysis methods (labelled 'instrument').

One amendment to this list are five (early) SALT papers published in the South African Journal of Science which is not listed in ADS. On 1 Oct 2015 there were 121 papers published. For a full list see http://astronomers.salt.ac.za/data/publications (collated by S. Bennett).

\section{Statistical evaluation}

A valuable and easily quantified statistic for the productivity of a telescope is the publication rate ([1]). Figure 1 shows the publication rate for several major observatories since start of their science operations. SALT-11 (in red) refers to the start of the full science operations in 2011. The open red circle and dotted line refer to the extrapolated number for 2015. The bottom panel shows the same, but normalised by the annual operating costs in million dollars (for details on the calculation of these numbers, see [4]). The cost effectiveness of SALT science is striking.

The citation frequency for 116 papers (i.e., excluding the five not listed in ADS) is shown in the left-hand panel of Fig. 2, while the right-hand panel gives the citation rate per paper. The spike 


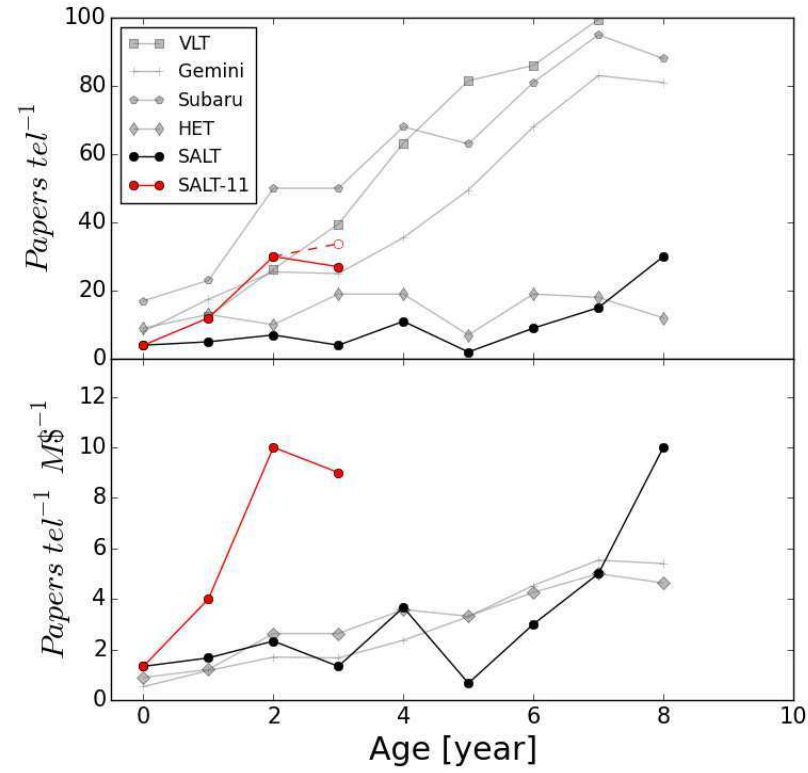

Figure 1: The publication rate for several major observatories since start of science operations (top) and normalised by annual operation costs (bottom).

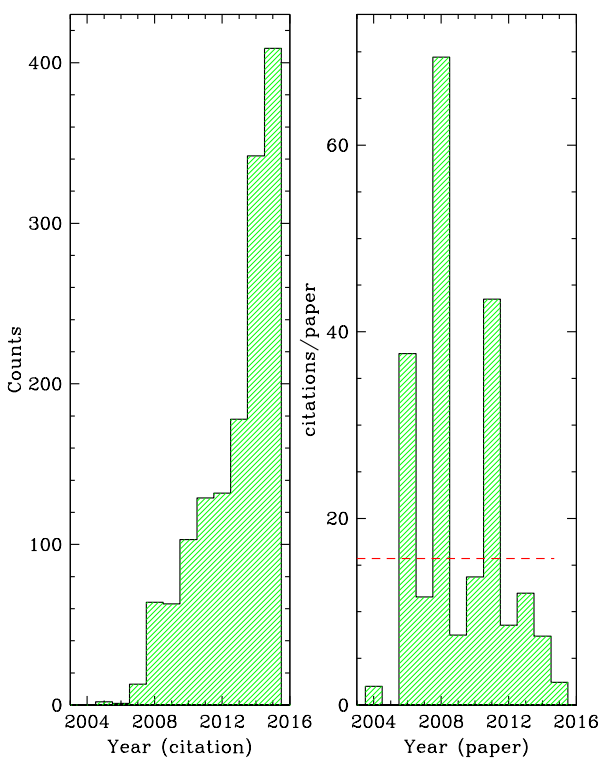

Figure 2: The citation frequency per year as derived from ADS, left, and the number of citations per paper per year, right.

in 2008 is due to two papers by the SDSS SN-survey consortium. The average citation per paper up to 2014 is 15.7 (dashed line) which compares reasonably well with the 20 citations per paper of other $>7 \mathrm{~m}$-class telescopes ([2]) considering the fact that SALT has not reached full productivity yet and that the citation rate becomes only meaningful more than two years after publication.

The 121 publications can be analysed in various ways. In the following, we make a distinction between statistics referring to the publications per se and statistics referring to the proposals that led to these publications.

\subsection{Statistics per publication}

There are 121 refereed SALT publications between 1995 and 1 Oct 2015, with 6 papers (on telescope and instruments) published before 'first light' in 2005. Of the total, 103 papers (85\%) use SALT observations (including 'first light' data from 2005), 7 (6\%) present general science projects using SALT, and $11(9 \%)$ describe the telescope or instrument.

Comparing the publication date with the observing dates of the published data, we find a $\sim 2$ year delay (left-hand panel in Fig. 3). The gap in 2009/2010 is due to the refurbishing of the telescope. Some papers are based on observations of more than one semester, hence the right-hand panel shows all observing semesters (mid- and end-year, respectively) versus publication date, where older semesters in a publication are shown as open circles. The mean and 1-sigma error for each publication year are shown in red. Only 2010 and 2013-2015 have 10 or more data points. The black line is for comparison: it would mean that the paper is published in the same year as when the observations were made.

Figure 4 shows histograms of some interesting statistics per publication. The top left panel shows that the partner affiliation of the 1st author is dominated by RSA (3) and OTHERS (4). 

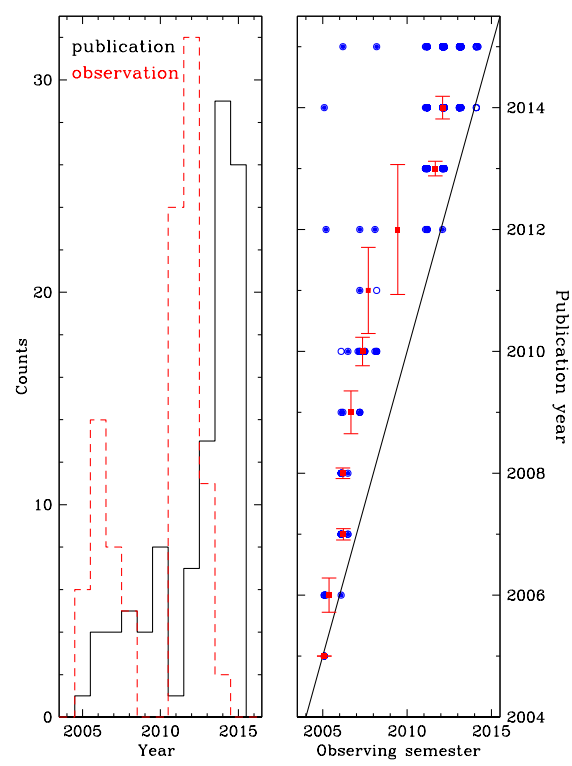

Figure 3: A comparison of publication date with observing semester of the published data. See text for details.
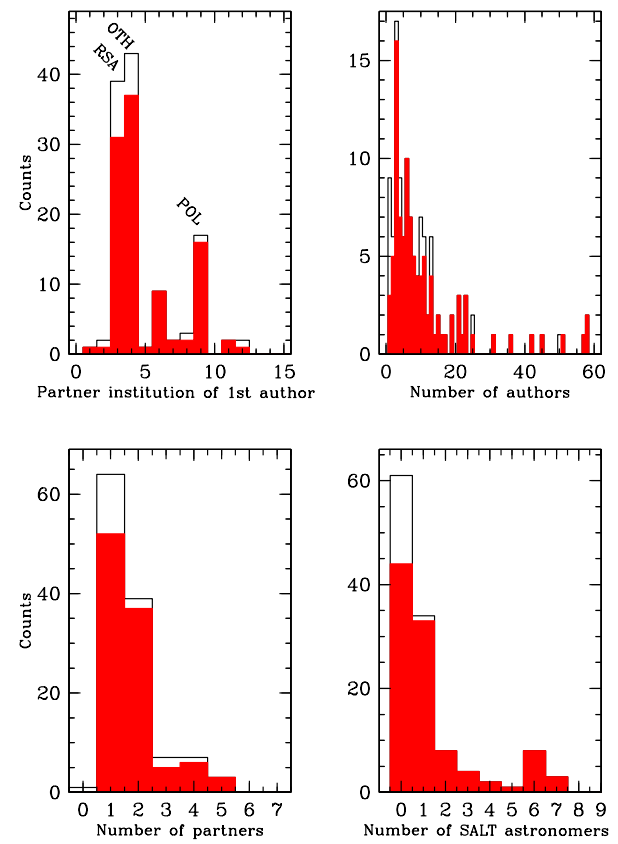

Figure 4: Statistics on publications. Filled histograms present data papers, open histograms present general science and instrument papers.

The top right panel shows that the majority of papers was published by small collaborations (the histogram is truncated: the two highest author numbers are 106 and 101). The bottom left panel shows the total number of SALT partner affiliations on the publication, and the bottom right panel shows the number of SALT Astronomers involved with the publication. The peak number of 6 occurred on publications in the years 2008-2010 where commissioning data was used and the SALT Astronomers were contributing significantly to ensure the quality of the data.

\subsection{Statistics per proposal}

The 103 publications presenting data are based on 121 proposals: 50 publications have used only one proposal, while in 19 cases data from 2 proposals were published, in 7 cases from 3 proposals, and in 3 cases from 4 proposals. Note that in 13 cases a proposal led to more than one publication. In addition, in 20 cases data sets from the Commissioning and Performance Verification time (2005/2006) were used, 2 publications made use of 'first light' observations, and one publication used data from the archive. For another 7 cases (after 2006), no proposal code existed (mainly from the interim time in 2007).

Of the 121 proposals used, 18 were 'Target of Opportunity' proposals and 15 proposals were explicitly used for student projects (16 PhD, $2 \mathrm{MSc}$ ). Note though that only the South African proposals explicitely required to tick the field in the proposal for student projects. We find that a total of 336 targets and 421 blocks (i.e., minimum schedulable unit) were published, resulting in more that 1347000 seconds or 374 hours observing time (including overheads).

Figures 5 and 6 show various information on how the published data was obtained. The frequency of instrument modes (Fig. 5) is derived from all 103 publications presenting data and 


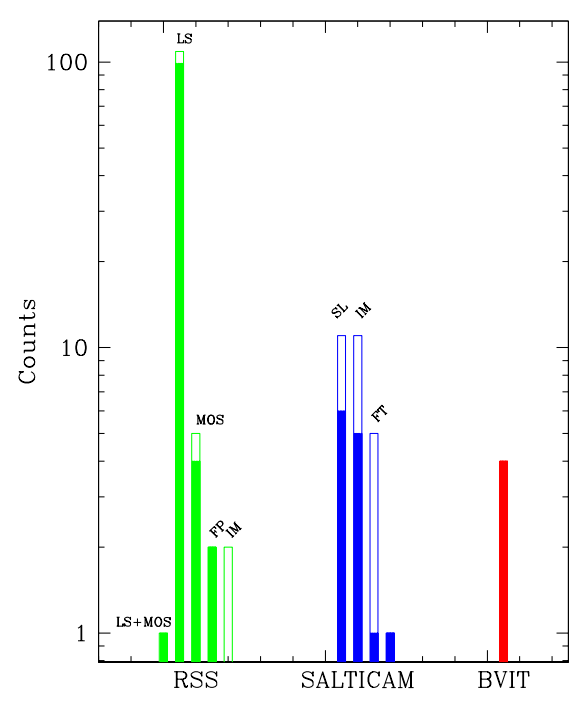

Figure 5: The use of instrument modes of all data papers in log scale (green: RSS; blue: SALTICAM; red: BVIT). For the open historgam no proposal information was available.
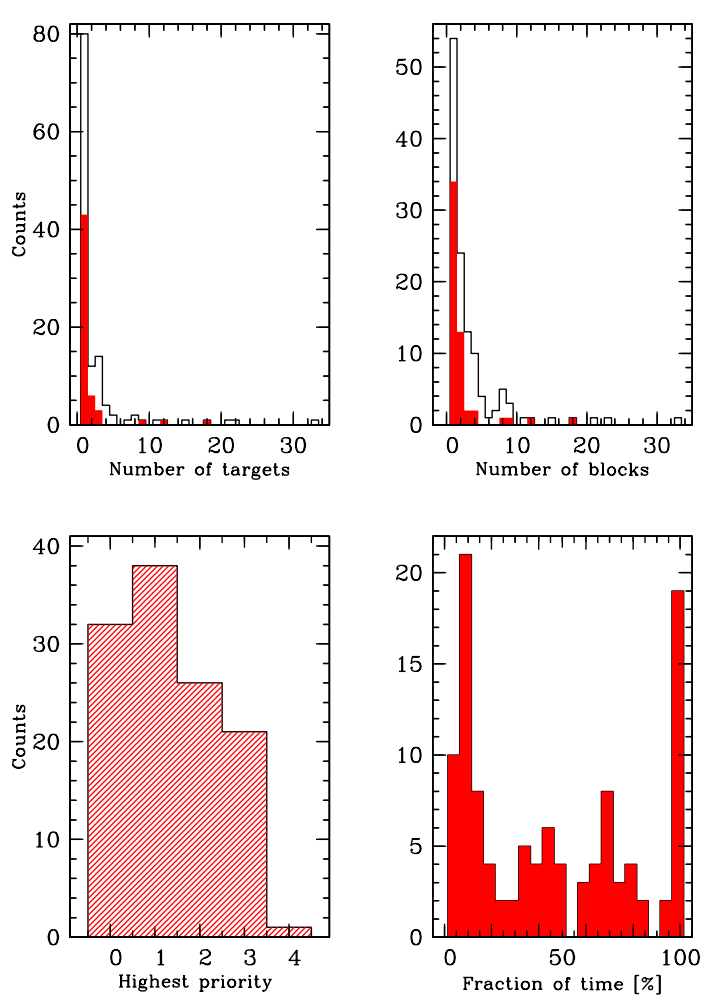

Figure 6: Statistics on 121 proposals. At the top, the filled histograms are based on data with fractional time $<20 \%$.

counted per proposal or, where no proposal was available (e.g., during commissioning), one entry per instrument was counted. The filled histogram presents data from the 121 proposals only. The majority of data was obtained with the Robert Stobie Spectrograph (RSS) in longslit mode, while BVIT (Berkeley Visible Image Tube) was rarely used. The RSS multi-object spectroscopy (MOS) and Fabry-Pérot (FP) imaging spectroscopy modes took longer to mature to routine operations, and they involve more complex data reductions.

The histograms in Fig. 6 were derived from the 121 proposals only. In 5 cases the proposal information was incomplete and we could not obtain priorities or fractional time information (i.e., time used in the paper with respect to the time spent on the proposal). The top panels in Fig. 6 show that most papers are based on one target or observing block only. That is somewhat reflected by the peak of small fractional times, see bottom right panel. The interpretation is that the most interesting objects are published first and before a survey is finished (see filled histograms for target and blocks where the fractional times are less than 20\%). It is expected that surveys usually need more time between observations and publication.

The bottom left panel shows the highest priority of the observed targets used in the paper (note that most proposals have targets with a variation of priorities). This partly reflects how well the TAC judges the proposed science with the caveat that high priorities tend to be observed first. A quick 
check though shows that the relative observing times on all proposals in 2012/13 are 1.0:2.8:4.5:5.1 for $\mathrm{P} 0: \mathrm{P} 1: \mathrm{P} 2: \mathrm{P} 3$, that is, a considerable fraction of time was indeed spent on priorities 2 and 3 . The bottom right panel shows the fractional times (i.e., time used in the paper with respect to the time spent on the proposal): There is an obvious bipolarity between small fractional times (e.g., a single object from a larger survey, see top panels) and full surveys / single-object proposals.

Finally, we can look at the publication efficiency per SALT partner. We counted, per partner, the proposals that have led to a publication (in total 133 proposals where 12 proposals were counted twice since the time was shared between two partners). In eight cases, Director's Discretionary Time (DDT) was used. We find that the top 4 partners using SALT share time in publications are South Africa (47.5\%), Poland (18\%), Dartmouth College (10.5\%), and the UK SALT Consortium (7.5\%). To calculate an efficiency, we divide this percentage by the SALT share time, which leads to the same top 4 partners but in different order: Poland and the UK Consortium published twice as much as expected by their SALT share time, South Africa 1.6 times, and Dartmouth College 1.2 times. All other partners have published less than expected by their SALT share time. Note though that the manpower varies widely across the partners, and that some partners concentrate more on larger long-term surveys not yet evident in these statistics. Furthermore, over-subscriptions (of P3), poorer weather and general degree of difficulty of programs also lead to partner usage statistics differing from expected.

\section{Conclusions}

We have analysed 121 refereed papers on SALT and SALT science that were published by 1 October 2015. The publication rate normalised for operations cost shows that four years into science operations SALT is one of the most cost effective observatories. Science operations proper started in 2011, and with a 2-year delay between observations and publication, we have about 2 years worth of unbiased statistics on the proposals (e.g., proposals from before 2011 were often incomplete). We find that these early science publications are dominated by single-target, longslit data which are easy to reduce. Often these targets were taken from larger (survey) proposals which shows that single objects in surveys are often interesting in their own right. The priorities determined by the TAC reflect the quality of the science output (with the caveat that not all low priority objects could be observed and the statistics are somewhat biased). We have also analysed the proposals that lead to publications by their SALT time charged and find that only four of the thirteen 13 SALT partners have published at a rate above that expected from their nominal share of observing time.

A further analysis of observing statistics (that is, weather statistics and observing efficiency) at SALT can be found in Crawford et al. [4].

\section{Acknowledgments}

This research has made use of NASA's Astrophysics Data System Bibliographic Services. PV and SMC acknowledge support from the National Research Foundation of South Africa. 


\section{References}

[1] Crabtree, D. R. 2015, Open Science at the Frontiers of Librarianship, 492, 90

[2] Abt, H. A. 2012, AJ 144, 91

[3] Rots, A. H., Winkelman, S. L., \& Becker, G. E. 2012, SPIE, 8448, 84480J

[4] Crawford, S. M., Koeslag, A., Romero Colmenero, E., et al. 2014, SPIE, 9149, 914909 